\title{
Special role of Foxp3 for the specifically altered microRNAs in Regulatory T cells of HCC patients
}

\author{
Long Chen ${ }^{1 \dagger}$, Huiying $\mathrm{Ma}^{1 \dagger}$, Heng Hu${ }^{1 \dagger}$, Lingling Gao ${ }^{1}$, Xuan Wang ${ }^{1}$, Jiaqi Ma ${ }^{1}$, Qiang Gao², Binbin Liu², \\ Guomin Zhou ${ }^{1}$ and Chunmin Liang ${ }^{1,2^{*}}$
}

\begin{abstract}
Background: Regulatory $T$ cells (Tregs) exhibit functional abnormalities in the context of hepatocellular carcinoma (HCC). The microRNAs (miRNAs) are identified as the key modulators in Tregs. This study was to explore whether the expression profiles of miRNAs of Tregs were different in HCC-activated Tregs and whether Foxp3 had special effects on them.

Methods: We isolated HCC-activated Tregs from mice bearing HCC and compared the expression profiles of miRNAs between HCC-activated Tregs and control Tregs by microarray. RNA interference against Foxp3 was also performed through transfection of synthetic siRNAs to Tregs for analyzing the effect of Foxp3 on the expression of miRNAs. Tregs isolated from HCC patients $(n=12)$ and healthy controls $(n=7)$ were used for validation of the differentially expressed miRNAs. Finally, bioinformatic analysis was applied to infer their possible roles.

Results: We found nine specifically altered miRNAs in HCC-activated Tregs from the murine model. After transfection with siRNAs against Foxp3, control Tregs showed obvious reduction of Foxp3 and five miRNAs were significantly changed; HCC-activated Tregs exhibited a slight reduction of Foxp3 with three miRNAs significantly changed. Tregs from HCC patients and healthy controls finally confirmed the up-regulation of four miRNAs (hsa-miR-182-5p, hsa-miR-214-3p, hsa-miR-129-5p and hsa-miR-30b-5p). Following bioinformatic analysis suggested these altered miRNAs would target eight important signaling pathways that could affect the functions of Tregs.

Conclusions: Our studies provided the first evidence that Tregs in HCC had the specifically altered expression of miRNAs, which was affected by Foxp3. These results are useful both in finding new biomarkers and in further exploring the functions of Tregs in HCC patients.
\end{abstract}

Keywords: Regulatory T cells, Hepatocellular carcinoma, microRNAs array, Foxp3 RNA interference, Bioinformatics

\section{Background}

Hepatocellular carcinoma (HCC) is the fifth most common cancer with relatively poor overall survival worldwide [1]. Accumulating evidence implies that $\mathrm{CD} 4^{+}$ $\mathrm{CD}_{2} 5^{+}$Foxp $^{+}$regulatory $\mathrm{T}$ cells (Tregs) are the critical factor affecting the progression and prognosis of $\mathrm{HCC}$ $[2,3]$. HCC patients have increased Tregs in peripheral blood, ascites and tumor tissue $[4,5]$ and this prevalence

\footnotetext{
* Correspondence: cmliang@fudan.edu.cn

${ }^{\dagger}$ Equal contributors

'Lab of Tumor Immunology, Department of Anatomy and Histology \& Embryology, Shanghai Medical College of Fudan University, 138 Yixueyuan Road, 200032 Shanghai, PR China

${ }^{2}$ Liver Cancer Institute, Zhongshan Hospital, Shanghai Medical College; Key laboratory of Carcinogenesis and Cancer Invasion, Ministry of Education, Fudan University, 136 Yixueyuan Road, 200032 Shanghai, PR China
}

of Tregs correlates with tumor stage and patients survival [6-8]. Not only the number but also the functional phenotypes of Tregs are found abnormal in HCC. Tregs in peripheral blood of HCC patients preferentially upregulate CCR6, which facilitates their migration to tumor sites [9]. HCC-activated Tregs also express high levels of glucocorticoid-induced tumor necrosis factor receptor (GITR) and the inducible T cell co-stimulator (ICOS), both of which are key mediators for the suppressive function of Tregs [10]. In addition, the enhanced suppressive function of Tregs is confirmed by different studies in HCC patients [10-12].

Tregs constitute the key components in tumor immune suppression [13]. Recent studies demonstrate that Tregs are widely modulated by the single-stranded microRNAs 
(miRNAs) $[14,15]$. Depleting Dicer, a key enzyme in the maturation of miRNAs, causes diminished Tregs and compromises their suppressive function [16]. Following studies demonstrate that miR-21 enhances the expression of Foxp3 while miR-31 suppresses it in human Tregs [17]. miR-155, modulated by Foxp3, guarantees competitive fitness of Tregs to the IL-2 signal by targeting suppressor of cytokine signaling 1 (SOCS1) protein $[18,19]$. Deficiency of miR-146a, another recently confirmed microRNA in Tregs, leads to increased Tregs with an impaired function [20].

However, it is still unclear at present for the following questions in liver cancer research. Firstly, it is still unknown that whether the expression profiles of miRNAs of Tregs are different between control Tregs and HCCactivated Tregs. Secondly, it is worthy to demonstrate whether Foxp3 has a special role and influences the expression profiles of miRNAs of Tregs in HCC. Thirdly, if there are any specifically altered miRNAs in Tregs in HCC, do they affect the functions of Tregs in HCC? It is also need to be further explored. In this study, we used Tregs from both the murine HCC model and HCC patients to answer these questions. We found nine miRNAs differentially expressed in HCC-activated Tregs and alterations of these miRNAs were specific to HCCactivated Tregs in the murine model. Foxp3 affected the expression of these miRNAs. Tregs isolated from human blood confirmed that four miRNAs up-regulated in HCC patients. To our knowledge, this study was the first attempt to characterize miRNAs perturbations and the consequences in Tregs activated by HCC. Our data provided a systemic view on alterations in $\mathrm{HCC}$-activated Tregs, which was not only useful in finding new biomarkers but also in further exploring the functions of Tregs in HCC patients.

\section{Methods}

\section{Human Tregs separation}

Peripheral blood mononuclear cells (PBMCs) were isolated by density gradient sedimentation using Lymphosep (Biowest, France) lymphocyte separation media. CD4 ${ }^{+}$ $\mathrm{CD}^{2} 5^{+} \mathrm{CD} 127^{-}$Tregs were enriched by human regulatory $\mathrm{T}$ cell isolation kit (Miltenyi, German). In brief, non-CD4 ${ }^{+}$ and $\mathrm{CD} 127^{\text {high }}$ cells were first depleted with microbeads and then the pre-enriched $\mathrm{CD} 4^{+} \mathrm{CD} 127^{\mathrm{dim}}$ cells went through positive selection for $\mathrm{CD} 25^{+} \mathrm{T}$ cells. The purity of Tregs was monitored via fluorescence-activated cell sorting (FACS). The participants included HCC patients $(n=12)$ and matched healthy controls $(n=7)$. Ethical approval for the use of human subjects was obtained from the Research Ethics Committee of Zhongshan Hospital (Shanghai, PR China), and informed consent was obtained from each participant.

\section{Cell lines and animals}

Hepa 1-6 (CRL-1830), the murine HCC cell line, was obtained from American Type Culture Collection (ATCC, USA) and maintained in DMEM (Biowest) supplemented with $10 \%$ FBS (Biowest). C57BL/6 J mice (6 to 8 weeks of age) were purchased from the Chinese Academy of Science and housed at the Animal Maintenance Facility of the Shanghai Medical College, Fudan University. All animal experiments were performed in conformity with the National Institutes of Health Guide for the Care and Use of Laboratory Animals. The Institutional Care of Experimental Animals Committee of Fudan University approved all animal protocols (Permit Number: SYXK 2009-0082).

Hepa 1-6 tumor bearing mice were established as following: mice were injected subcutaneously at the left flank with $3 \times 10^{6}$ Hepa $1-6$ cells in $200 \mu \mathrm{L}$ RPMI 1640 (Biowest) or $200 \mu \mathrm{L}$ RPMI 1640 alone as control. Three groups of tumor bearing mice and control mice were established (12 mice in each group). Two weeks after inoculation, the mice with visible tumor were sacrificed and spleens were collected for isolation of Tregs using mouse regulatory $\mathrm{T}$ cell isolation kit (Miltenyi). In brief, $\mathrm{CD}^{+} \mathrm{T}$ cells were enriched by negative selection and then went through positive selection for $\mathrm{CD} 25^{+} \mathrm{T}$ cells. The purity of Tregs was monitored via FACS.

\section{FACS analysis}

For FACS, previously collected Tregs were stained with regulatory $\mathrm{T}$ cell Kit (eBioscience, USA). Briefly, cells were first incubated with anti-CD4-FITC and antiCD25-PE Abs at $4^{\circ} \mathrm{C}$ for 30 minutes. After washing, cells were treated with fixation/permeabilization buffer and then incubated with anti-Foxp3-PE-Cy5 Abs at $4^{\circ} \mathrm{C}$ for 30 minutes. Stained cells were subsequently analyzed using an EPICS ALTRA Flow Cytometer (Beckman Coulter, USA).

\section{Transfection of siRNAs}

Validated siRNAs against mouse Foxp3 and AllStars negative control siRNAs were obtained from Qiagen company in a FlexiTube format (Qiagen, German). Four species of siRNAs against Foxp3 (SI01005319, SI01005326, SI01005333 and SI01005340) were mixed in equal amount to generate the Foxp3 siRNAs pool. siRNAs were transfected into HCC-activated Tregs and control Tregs using HiPerFect transfection reagent (Qiagen) according to the manufacturer's protocol. Tregs were maintained in RPMI 1640 supplemented with $10 \%$ heat-inactivated FBS, $5 \mu \mathrm{g} / \mathrm{mL}$ plate-bounded anti-CD3, $5 \mu \mathrm{g} / \mathrm{mL}$ soluble anti-CD28 antibodies (Biolegend, USA) and $40 \mathrm{ng} / \mathrm{mL} \mathrm{rm} \mathrm{IL-2}$ (PeproTech, USA) at $37^{\circ} \mathrm{C}$ in humidified atmosphere containing $5 \% \mathrm{CO}_{2}$ in air. Mixture of $100 \mathrm{nM}$ of siRNAs and $6 \mu \mathrm{L}$ of 
HiPerFect reagent were used for each transfection. Then Tregs were harvested for further analysis fortyeight hours after transfection. Two-step qRT-PCR and FACS validated the efficiency of Foxp3 RNA interference (RNAi).

\section{miRNAs microarray}

miRNAs microarray was assisted by Kangcheng Bioscience Service Company (Shanghai). In brief, total RNA was extracted from Tregs of each group using TRIzol (Invitrogen, USA) and miRNeasy mini kit (Qiagen). RNA quality and quantity was measured by NanoDrop-1000 spectrophotometer (Nanodrop Technologies, USA). miRNAs were labeled by the miRCURY ${ }^{\mathrm{TM}} \mathrm{Hy}^{\mathrm{Ts}} / \mathrm{Hy}^{\mathrm{mm}}$ Power labeling kit (Exiqon, Denmark) and hybridized on the miRCURYTMLNA Array (v.16.0) (Exiqon). The GenePix 4000B microarray scanner (Molecular Devices, USA) scanned the slides. After normalization, volcano plot was used to identify the differentially expressed miRNAs (fold change $\geq 1.5$ and $P$-value $\leq 0.05$ fold change: the ratio of normalized intensities, HCC-activated Tregs vs. control Tregs; $P$-value: $t$-test results between groups). Three independent arrays were performed for $\mathrm{HCC}$-activated Tregs and control Tregs. One array was performed for HCCactivated Tregs transfected with siRNAs against Foxp3 or control siRNAs respectively; one array was performed for control Tregs transfected with siRNAs against Foxp3 or control siRNAs respectively.

\section{Real-time PCR}

Total RNA was extracted from Tregs using TRIzol (Invitrogen) and miRNeasy mini kit (Qiagen). RNA quality and quantity was measured by NanoDrop-1000 spectrophotometer. miRNAs were transcribed to cDNA by cDNA Synthesis Kit (Epicentre, USA) with specific reverse transcription primers (The primers were listed in Additional file 1: Table S1 ). Then the cDNA was used for real time-PCR by miScript SYBRGreen PCR Kit (Qiagen) with specific primers (The primers were listed in Additional file 2: Table S2). For quantification of Foxp3, the cDNA was first synthesized by cDNA Synthesis Kit with Oligo $(\mathrm{dT})_{21}$ primer, and the real timePCR was performed by miScript SYBRGreen PCR Kit with specific primers (Invitrogen, the primers were listed in Additional file 2: Table S2. The expression levels of miRNAs were presented as $2^{-\Delta \Delta} \mathrm{Ct}$ or $2^{-\Delta \mathrm{Ct}}$ relative to U6 levels and the levels of mRNA were presented as $2^{-\Delta \Delta C t}$ relative to GAPDH levels.

\section{Bioinformatic analysis}

We predicted target genes using the online algorithm TargetScan (Release 6.2, http://www.targetscan.org) [21]. The network of miRNAs and target genes was constructed in the software Exploratory Gene Association
Networks (EGAN) [22]. In brief, the total target genes lists were imported into EGAN and further filtered based on Tregs MeSH term (T-Lymphocytes, regulatory). Target genes with direct relation with Tregs $\mathrm{MeSH}$ term were presented in the network and further analyzed for functional enrichment. Pathway enrichment was based on the pathways database Kyoto Encyclopedia of Genes and Genomes (KEGG, http://www.genome.jp/ kegg/) through DAVID Bioinformatics Resources 6.7 (http://david.abcc.ncifcrf.gov/home.jsp) [23].

\section{Statistical analysis}

Microarray data of miRNAs was analyzed by volcano plot and the filtering criteria were set at expression fold change $\geq 1.5$ and $P$-value $\leq 0.05$. Unsupervised hierarchical clustering was performed by MultiExperiment Viewer software (v4.8.1, USA). qRT-PCR validation data was analyzed by Mann-Whitney test. Statistical significance was set at $P$-value $<0.05\left(^{*}\right)$ or $P$-value $<0.01{ }^{(* *)}$.

\section{Results \\ miRNAs were differentially and specifically expressed in HCC-activated Tregs}

Tregs were isolated from spleens of mice and the purity of Tregs was examined by fluorescence-activated cell sorting (FACS); only the cells with the purity above 95\% (data not shown) were further processed for microarray analysis. Differentially expressed miRNAs in HCC-activated Tregs were selected by volcano plot filtering (fold change $\geq 1.5$ and $P$-value $\leq 0.05$ ). Eleven miRNAs were identified as shown in Figure 1A. There were four up-regulated miRNAs (mmu-miR-709, mmumiR-467a-3p, mmu-miR-182-5p and mmu-miR-25-5p) and seven down-regulated miRNAs (mmu-miR-615-3p, mmu-miR-409-3p, mmu-miR-680, mmu-miR-129-5p, mmumiR-151-5p, mmu-miR-142-5p and mmu-miR-30b-5p), as the values presented in Table 1 . Then we performed unsupervised hierarchical clustering of the eleven miRNAs. We found these miRNAs clearly discriminated the HCC-activated Tregs from control Tregs, as shown in Figure 1B.

By TargetScan, we found that mmu-miR-25-5p, mmumiR-615-3p, mmu-miR-151-5p and mmu-miR-680 had few target genes directly relating with Tregs in $\mathrm{MeSH}$ database, so we excluded the four miRNAs for further exploration. As mmu-miR-155 and mmu-let-7i have been well documented in T cells [19,24,25], we observed that mmu-miR-487b-5p and mmu-miR-214-3p were classified into the same group with mmu-miR-155 and mmu-let-7i respectively after hierarchical clustering (data not shown). Therefore, we also included these two miRNAs for further validation. To verify the credibility of qRT-PCR validation, we included the miR-344e-5p as a negative control as it did not pass volcano plot filtering (fold change $=1.85$, 




$P$-value $=0.54)$ in microarray. Among the ten miRNAs validated by qRT-PCR, we found that $m m u-m i R-487 b$ 5p, mmu-miR-709, mmu-miR-182-5p, mmu-miR-214-3p and $m m u-m i R-467 a-3 p$ were up-regulated in HCCactivated Tregs, $m m u-m i R-142-5 p, m m u-m i R-30 b-5 p$, $m m u-m i R-409-3 p$ and $m m u-m i R-129-5 p$ were down- regulated $(P<0.01)$, while miR-344e-5p did not change significantly, as shown in Figure $1 \mathrm{C}$.

Foxp3 was involved in regulating the miRNAs

As Foxp3 is the master regulator in Tregs, it prompts us to check whether these miRNAs would be specifically 
Table 1 Differentially expressed miRNAs in HCC-activated Tregs

\begin{tabular}{ccc}
\hline Name & Fold change (Tumor vs. Control) & $P$-value \\
\hline mmu-miR-25-5p & 2.21 & 0.04 \\
mmu-miR-709 & 1.98 & 0.02 \\
mmu-miR-467a-3p & 1.82 & 0.04 \\
mmu-miR-182-5p & 1.54 & 0.05 \\
mmu-miR-129-5p & 0.29 & 0.02 \\
mmu-miR-680 & 0.34 & 0.02 \\
mmu-miR-615-3p & 0.36 & 0.00 \\
mmu-miR-409-3p & 0.44 & 0.02 \\
mmu-miR-30b-5p & 0.51 & 0.05 \\
mmu-miR-151-5p & 0.61 & 0.03 \\
mmu-miR-142-5p & 0.63 & 0.04 \\
\hline
\end{tabular}

affected by Foxp3. We compared the mean fluorescence intensity (MFI) of Foxp3 in Tregs after magnetic sorting. FACS results showed that the sorted Tregs had comparable percentages of Foxp3 positive cells; however, the MFI was higher in HCC-activated Tregs compared with control Tregs (Figure 2A). We transfected Tregs with siRNAs against Foxp3 or negative controls and fortyeight hours we determined the efficiency of silencing. The qRT-PCR data demonstrated that mRNA levels of Foxp3 reduced significantly (34\%) in control Tregs, whereas the levels did not change significantly in HCCactivated Tregs (Figure 2B, left). While the FACS results showed the protein levels of Foxp3 reduced in both groups, we observed a more potent decrease of Foxp3 in control Tregs after siRNA silencing (Figure 2B, right). Then we examined the expression levels of the nine miRNAs in Tregs from our microarray data after transfection. In control Tregs, mmu-miR-487b-5p, mmu-miR214-3p, mmu-miR-30b-5p and mmu-miR-129-5p showed significant down-regulation while mmu-miR-409-3p showed significant up-regulation (Figure $2 \mathrm{C}$, left). Compared with control Tregs, although mmu-miR-487b-5p and $m m u-m i R-129-5 p$ showed similar down-regulation in HCC-activated Tregs, mmu-miR-409-3p was actually significantly down-regulated; $m m u-m i R-214-3 p$ and $m m u-m i R-30 b-5 p$ did not exhibit significant changes (Figure 2C, right).

\section{Expression patterns of the miRNAs in human Tregs}

We wondered whether these miRNAs were also differentially expressed in HCC patients. Because miR-487b5p, miR-709 and miR-467a-3p did not express in human tissue (miRBase 19), we checked the expression levels of the rest six miRNAs in Tregs from peripheral blood samples. Compared with the healthy controls, the expression levels of hsa-miR-182-5p, hsa-miR-214-3p, hsa-miR-129$5 p$ and $h s a-m i R-30 b-5 p$ were significantly up-regulated in
Tregs from HCC patients while the hsa-miR-409-3p and $h s a-m i R-142-5 p$ did not show significant changes (Figure 3).

\section{Possible roles of target genes inferred by bioinformatic analysis}

The functions of these four miRNAs (hsa-miR-182-5p, hsa-miR-214-3p, hsa-miR-129-5p and hsa-miR-30b-5p) in human Tregs are not clear. Therefore, we applied bioinformatic methods to explore their roles. Target genes of these four miRNAs were predicted by TargetScan 6.2 and all the genes were imported to software EGAN. The total 109 target genes involved in Tregs functions were enriched based on $\mathrm{MeSH}$ database and constructed into the network (Figure 4A). These target genes were further analyzed by functional enrichment based on KEGG pathways via DAVID and eight pathways were enriched with statistical significance (Figure 4B). Among these pathways, two pathways were involved in cytokine signaling (Cytokine-cytokine receptor interaction and Jak-STAT signaling pathway); two pathways were associated with chemotaxis (Chemokine signaling pathway and Cell adhesion molecules (CAMs)); two pathways were related with immune response to graft (Allograft rejection and Graft-versus-host disease). The other two pathways were Intestinal immune network for IgA production and NOD-like receptor signaling pathway.

\section{Discussion}

In this study, we found nine differentially expressed miRNAs in Tregs from the murine HCC model, which were modulated by Foxp3, and validated four of them in Tregs from HCC patients. Bioinformatic analysis suggested the four miRNAs had important roles by targeting genes in several pathways affecting Tregs functions.

It has been reported that alterations of miRNAs in $\mathrm{CD}^{+}{ }^{+} \mathrm{T}$ cells and Tregs are correlated with certain activation states and diseases [26-28]. Our array data found a group of differentially expressed miRNAs in Tregs from the murine HCC model. Interestingly, the expression patterns of these miRNAs were specific to HCC-activated Tregs. It is supposed that Tregs suppress the immune response in a context dependent way [29-31]. For example, depleting the signal transducer and activator of transcription 3 (STAT3), which is essential for proper development of Th17 cells, results in failure of Tregs to suppress Th17 cell-mediated disease [32]. Assisted by bioinformatic analysis, we selected ten miRNAs for qRT-PCR validation. The validation data were consistent with array results, indicating the up-regulation of five miRNAs and down-regulation of four miRNAs. Because miRNAs can simultaneously regulate a large number of genes, they might be the proper candidate for this context dependent modulation. We proposed that the specific tumor antigen, 


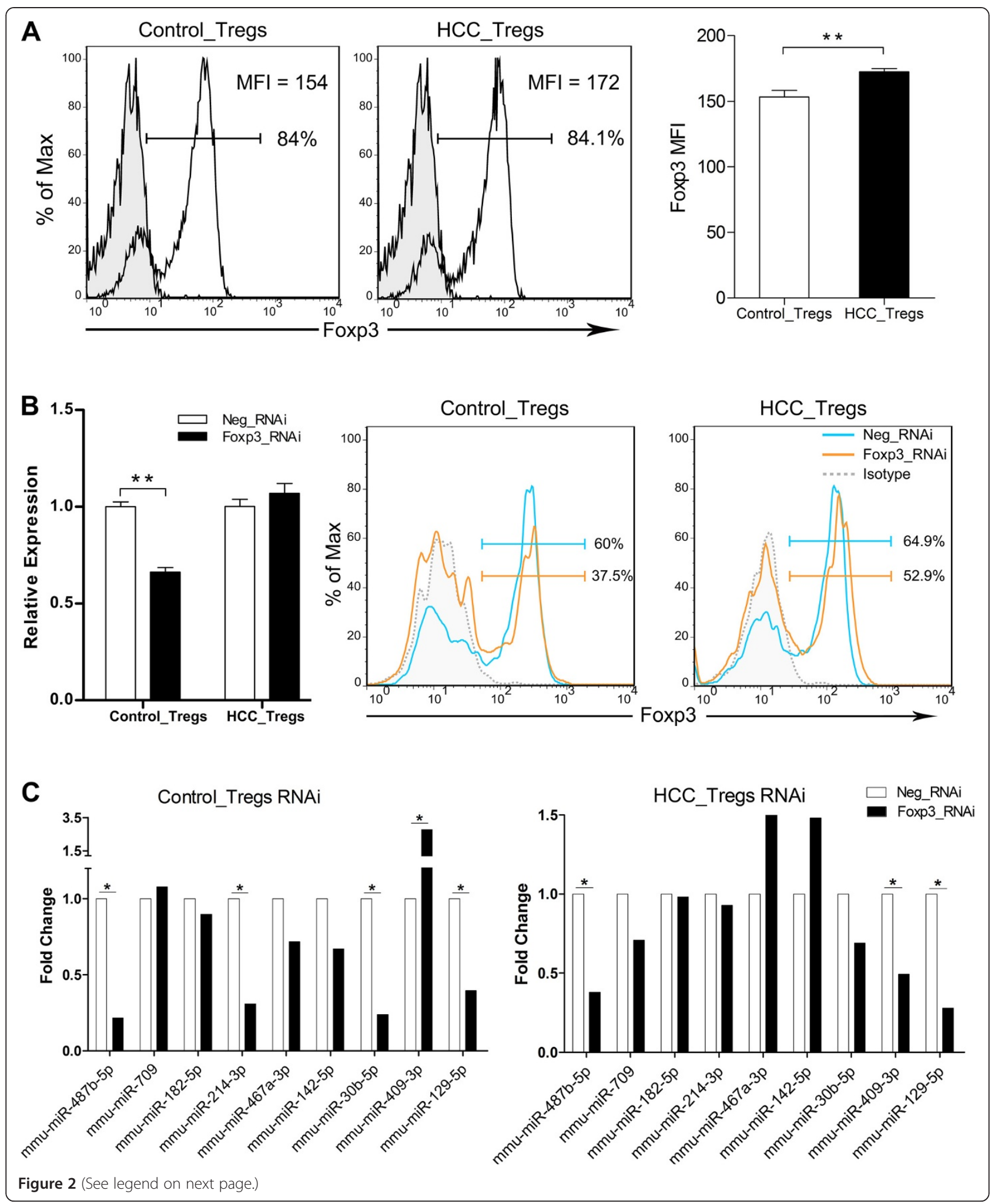


(See figure on previous page.)

Figure 2 Special modulation of the miRNAs by Foxp3. (A) Representative FACS plots of Foxp3 were shown in control Tregs and HCC-activated Tregs (left). Mean fluorescence intensity (MFI) was included in each of the panels. Summary of the Foxp3 MFI was presented (right) in control Tregs and HCC-activated Tregs. (B) Tregs were transfected with Foxp3-specific siRNAs or negative controls and forty-eight hours later cells were harvested. Expression levels of Foxp3 were determined by qRT-PCR (left) and representative FACS plots were shown in control Tregs and HCC-activated Tregs (right). Results in (A) and (B) were presented as mean \pm SEM of three independent experiments performed in triplicates and analyzed by the two-tailed Student's t-test. ${ }^{*} P<0.01$ for indicated comparison. (C) Expression patterns of the nine miRNAs after Foxp3 silencing in control Tregs (left) and HCC-activated Tregs (right). The expression levels of each miRNAs after Foxp3 silencing were shown as fold change relative to the negative control siRNAs. Data were from one microarray. ${ }^{*}$ fold change $>1.5$ for indicated comparison. Control_Tregs: Tregs from control mice; HCC_Tregs: Tregs from mice bearing Hepa 1-6; Neg_RNAi: Tregs transfected with negative control siRNAs; Foxp3_RNAi: Tregs transfected with siRNAs against Foxp3.

tumor associated antigen or tumor derived signals might contribute to the unique alterations in HCC-activated Tregs, which was possibly mediated by miRNAs.

Because Foxp3 is the crucial transcription factor in Tregs, we wondered whether these altered miRNAs were affected by Foxp3. As Foxp3 has been reported to be upregulated in activated Tregs in some reports [33,34], we first compared the MFI of Foxp3 in control Tregs and HCC-activated Tregs. Consisted with previous reports, we found higher MFI in HCC-activated Tregs. After transfection of siRNAs against Foxp3, we determined the mRNA and protein levels of Foxp3. Control Tregs showed significant down-regulation of Foxp3 after silencing at both the mRNA and protein levels; however, HCC-activated Tregs showed slight down-regulation of Foxp3 protein, and no significant changes of Foxp3 mRNA. So the increased expression of Foxp3 in HCC- activated Tregs might involve in the reduced efficacy of Foxp3 RNAi. One report recently demonstrates that under certain inflammatory milieu Foxp3 undergoes phosphorylation, which affects its stability and function [35]. This report and our present data support the hypothesis that both the modification state and the expression level of Foxp3 in HCC-activated Tregs can affect the efficiency of Foxp3 silencing.

We also found five miRNAs showed significant fold changes after Foxp3 RNAi in control Tregs, among which three miRNAs showed significant fold change in HCCactivated Tregs. Two miRNAs (mmu-miR-214-3p and $m m u-m i R-30 b-5 p$ ) were significantly changed only in control Tregs. Our findings were consistent with previous studies, which have demonstrated that Foxp3 modulates the expression of miR-155 that maintains the functions of Tregs [18]. Considering the relatively low silencing efficacy

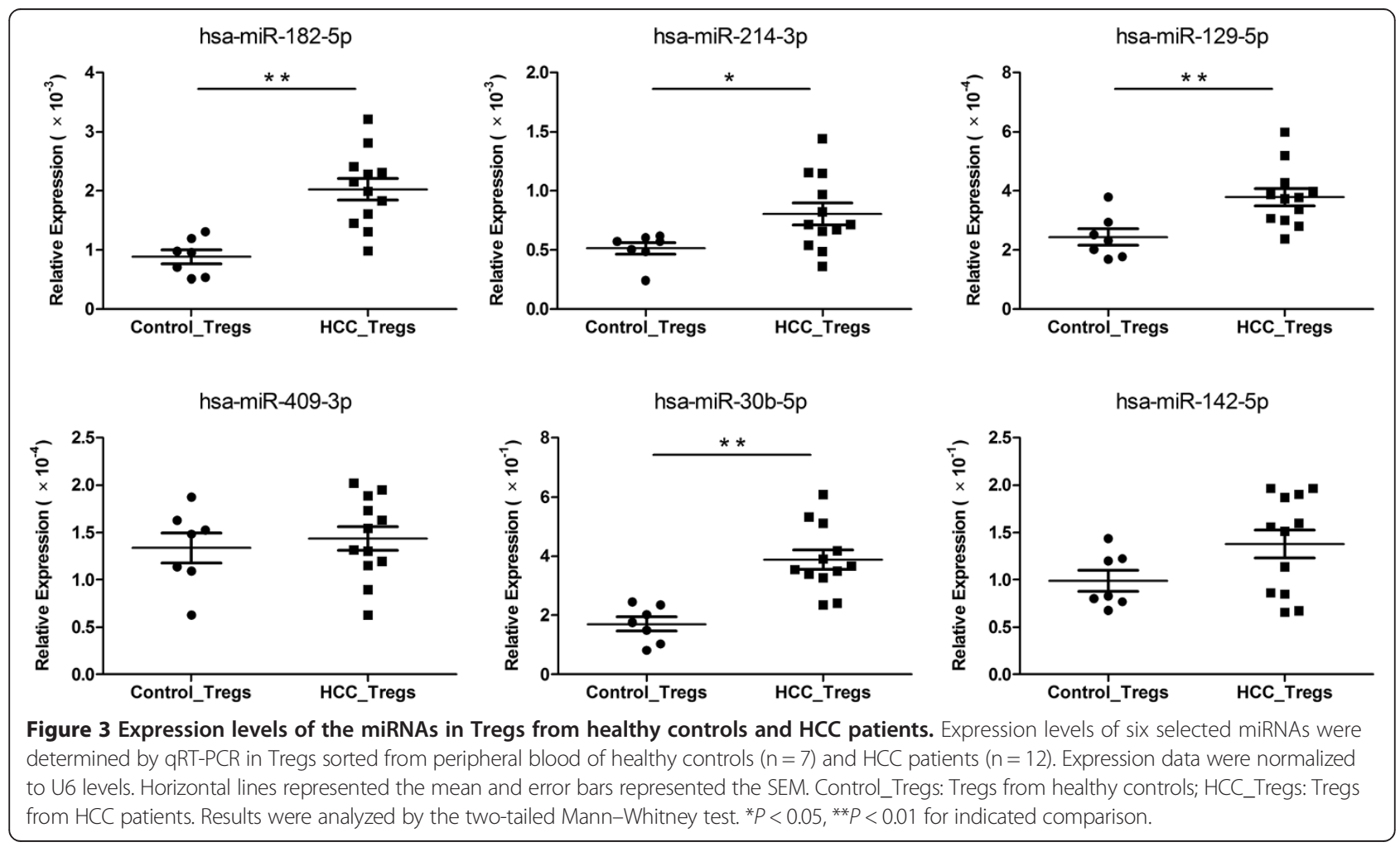



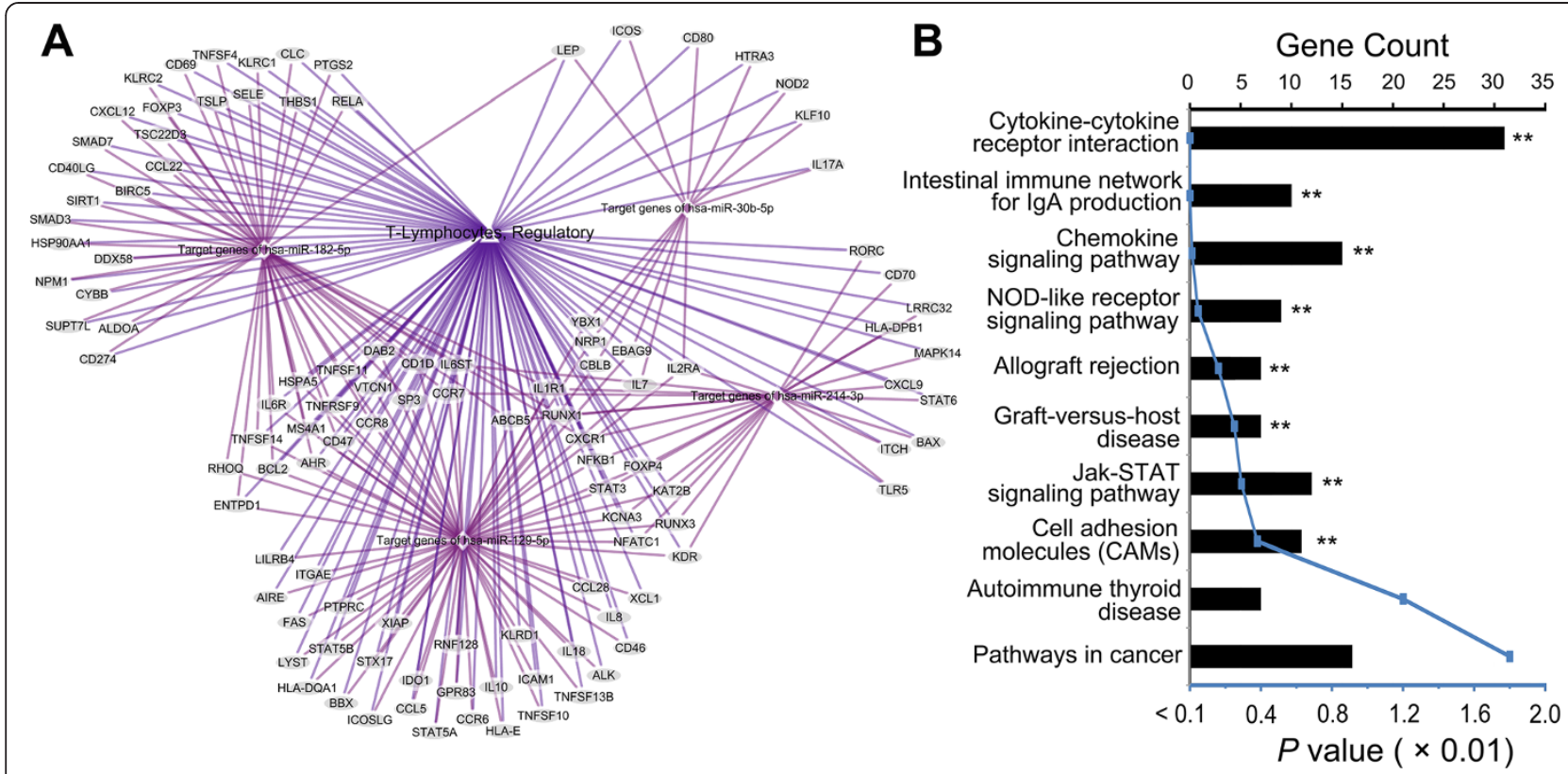

Figure 4 Bioinformatic analysis of target genes of the four miRNAs. (A) The network of target genes of the four miRNAs involved in Tregs functions. Each ellipse represented a target gene predicted by TargetScan. The four rhombuses were miRNAs and the triangle was the MeSH term of Tregs. Purple lines indicated genes involved in Tregs according to MeSH database; magenta lines outlined the miRNAs-target genes relations. (B) Pathway enrichment of target genes. The y-axis represented pathways enriched based on predicted target genes of the four miRNAs. The histogram indicated gene number in each pathway (upper black $x$-axis); the curve line indicated the $P$ value (lower blue $x$-axis). ${ }^{*} P<0.01$ with the Bonferroni correction.

of Foxp3 in HCC-activated Tregs, we thought the modulation of these two miRNAs were not so sensitive to Foxp3 levels compared with that of the other three miRNAs.

We further validated the expression levels of these miRNAs in Tregs from HCC patients and healthy controls. Because miR-487b-5p, miR-709 and miR-467a-3p did not express in human tissue (miRBase 19), we validated the expression levels of the rest six miRNAs. Four miRNAs showed significant changes in HCC-activated Tregs compared with healthy controls. Interestingly, compared with data from the murine model, two of the four miRNAs (hsa-miR-182-5p and hsa-miR-214-3p) showed the similar up-regulation while the other two miRNAs ( $h s a-m i R-129-5 p$ and $h s a-m i R-30 b-5 p$ ) showed reverse changes. We were not sure whether this discrepancy was due to the differences of species or HCC tumor models. Further experiments were need to clarify this question.

The functions of four up-regulated miRNAs were not reported in Tregs and we performed bioinformatic analysis to infer their possible roles. The target genes with direct relations with Tregs $\mathrm{MeSH}$ term were found significantly involved in eight pathways. Two of them were cytokine signaling, including genes IL6ST, IL6R, STAT3 and $I L 17 A$ which have been reported to facilitate the differentiation of Th17 by inhibiting Tregs induction [36-38]. Up-regulation of these miRNAs might break the balance between Th17 and Tregs and finally accelerate the production of Tregs, which contributes to the abnormal homeostasis of Tregs in HCC $[9,39,40]$. Another two pathways related with chemotaxis, which has been reported to be critical for the migration and distribution of Tregs in HCC. CC chemokine receptor 6 (CCR6) axis has an important role in recruiting Tregs to tumor sites in HCC [9]; TGF-beta and macrophage-derived chemokine (CCL22) signaling pathways induce aggregation of Tregs at the tumor sites in HCC too [41]. These two pathways included genes in chemotaxis and cell adhesion such as CCR6, CCR7, CXCR1, SELE and ICOS $[9,42-44]$. These alterations might contribute to the abnormal distribution of Tregs in HCC at the tumor sites. We also found two pathways relating with immune response to allograft (Allograft rejection and Graft-versus-host disease). Although it is well established that Tregs are critical for maintaining the tolerance to allograft [45-47], it is not clear whether the same genes or pathways work similarly in Tregs during the progression of HCC. These new clues needed further exploring. IgA production is essential for the intestinal homeostasis, in which Tregs are indispensable via secretion of TGF-beta $[48,49]$. It was possible that Tregs applied the same mechanism via TGF-beta in HCC. NOD-like receptor is one of the conserved patternrecognition receptors (PRRs) which included Toll-like receptors (TLRs) [50]. Previous studies have demonstrated 
that TLR1, TLR2, TLR4 and TLR7 have important functions in Tregs [51-54] and we proposed that NOD-like receptors were new key PRRs in the context of HCC.

\section{Conclusions}

In summary, we confirmed nine differentially expressed miRNAs in Tregs from the HCC murine model. These miRNAs exhibited a specific expression pattern in HCCactivated Tregs and were affected by Foxp3. Four miRNAs were finally found to be up-regulated in HCC patients for the first time. Bioinformatic analysis indicated the four miRNAs (hsa-miR-182-5p, hsa-miR-214-3p, hsa-miR-129$5 p$ and $h s a-m i R-30 b-5 p$ ) targeted eight signaling pathways involved in Tregs. These results provided interesting information on the intrinsic functional changes occurred in HCC-activated Tregs, which were worthy of further exploration.

\section{Additional files}

Additonal file 1: Table S1. Primers for Reverse Transcription.

Additional file 2: Table S2. Primers for Real-time PCR.

\section{Abbreviations}

HCC: Hepatocellular carcinoma; Tregs: Regulatory T cells; miRNAs: microRNAs; RNAi: RNA interference; MFI: Mean fluorescence intensity;

FACS: Fluorescence-activated cell sorting.

\section{Competing interests}

The authors declare that they have no competing interests.

\section{Authors' contributions}

LC, HM and $\mathrm{HH}$ performed the experiments, interpreted the findings and prepared the manuscript. LG, XW and JM assisted in animals' maintenance. QG prepared blood samples and obtained informed consent from the patients. BL performed the statistical analysis. GZ participated in the design, $\mathrm{CL}$ conceived of the study, participated in the design, and assisted with data interpretation and manuscript writing. All authors read and approved the final manuscript.

\section{Acknowledgements}

This work was supported by grants from the National Science Foundation of China (No.30871312) and the National Basic Research Program of China (973 program, No.2011CB910700).

Received: 7 February 2014 Accepted: 4 July 2014

Published: 7 July 2014

\section{References}

1. El-Serag HB: Hepatocellular carcinoma. N Engl J Med 2011, 365(12):1118-1127.

2. Zhou J, Ding T, Pan W, Zhu LY, Li L, Zheng L: Increased intratumoral regulatory $T$ cells are related to intratumoral macrophages and poor prognosis in hepatocellular carcinoma patients. Int J Cancer 2009, 125(7):1640-1648.

3. Wang HY, Wang RF: Regulatory T cells and cancer. Curr Opin Immunol 2007, 19(2):217-223.

4. Fu J, Xu D, Liu Z, Shi M, Zhao P, Fu B, Zhang Z, Yang H, Zhang H, Zhou C, Yao J, Jin L, Wang H, Yang Y, Fu YX, Wang FS: Increased regulatory T cells correlate with CD8 T-cell impairment and poor survival in hepatocellular carcinoma patients. Gastroenterology 2007, 132(7):2328-2339.

5. Gao Q, Qiu SJ, Fan J, Zhou J, Wang XY, Xiao YS, Xu Y, Li YW, Tang ZY: Intratumoral balance of regulatory and cytotoxic $T$ cells is associated with prognosis of hepatocellular carcinoma after resection. J Clin Oncol 2007, 25(18):2586-2593.

6. Shen X, Li N, Li H, Zhang T, Wang F, Li Q: Increased prevalence of regulatory $T$ cells in the tumor microenvironment and its correlation with TNM stage of hepatocellular carcinoma. J Cancer Res Clin Oncol 2010, 136(11):1745-1754.

7. Takata Y, Nakamoto Y, Nakada A, Terashima T, Arihara F, Kitahara M, Kakinoki K, Arai K, Yamashita T, Sakai Y, Mizukoshi E, Kaneko S: Frequency of CD45RO + subset in CD4 + CD25(high) regulatory T cells associated with progression of hepatocellular carcinoma. Cancer Lett 2011, 307(2):165-173.

8. Beyer M, Schultze JL: Regulatory T cells in cancer. Blood 2006, 108(3):804-811.

9. Chen KJ, Lin SZ, Zhou L, Xie HY, Zhou WH, Taki-Eldin A, Zheng SS: Selective recruitment of regulatory $T$ cell through CCR6-CCL20 in hepatocellular carcinoma fosters tumor progression and predicts poor prognosis. PLOS ONE 2011, 6(9):e24671.

10. Pedroza-Gonzalez A, Verhoef C, ljzermans JN, Peppelenbosch MP, Kwekkeboom J, Verheij J, Janssen HL, Sprengers D: Activated tumor-infiltrating CD4+ regulatory $T$ cells restrain antitumor immunity in patients with primary or metastatic liver cancer. Hepatology 2013, 57(1):183-194

11. Cao M, Cabrera R, Xu Y, Firpi R, Zhu H, Liu C, Nelson DR: Hepatocellular carcinoma cell supernatants increase expansion and function of CD4(+) CD25(+) regulatory T cells. Lab Invest 2007, 87(6):582-590.

12. Cabrera R, Ararat M, Eksioglu EA, Cao M, Xu Y, Wasserfall C, Atkinson MA, Liu C, Nelson DR: Influence of serum and soluble CD25 (sCD25) on regulatory and effector T-cell function in hepatocellular carcinoma. Scand I Immunol 2010, 72(4):293-301.

13. Sakaguchi S, Yamaguchi T, Nomura T, Ono M: Regulatory T cells and immune tolerance. Cell 2008, 133(5):775-787.

14. Gao Y, Lin F, Su J, Gao Z, Li Y, Yang J, Deng Z, Liu B, Tsun A, Li B: Molecular mechanisms underlying the regulation and functional plasticity of FOXP3(+) regulatory T cells. Genes Immun 2012, 13(1):1-13.

15. Xiao C, Rajewsky $\mathrm{K}$ : MicroRNA control in the immune system: basic principles. Cell 2009, 136(1):26-36.

16. Liston A, Lu LF, O'Carroll D, Tarakhovsky A, Rudensky AY: Dicer-dependent microRNA pathway safeguards regulatory T cell function. J Exp Med 2008, 205(9):1993-2004.

17. Rouas R, Fayyad-Kazan $H$, El Zein N, Lewalle $P$, Rothe F, Simion A, Akl H, Mourtada M, El Rifai M, Burny A, Romero P, Martiat P, Badran B: Human natural Treg microRNA signature: role of microRNA-31 and microRNA-21 in FOXP3 expression. Eur J Immunol 2009, 39(6):1608-1618.

18. Lu LF, Thai TH, Calado DP, Chaudhry A, Kubo M, Tanaka K, Loeb GB, Lee H, Yoshimura A, Rajewsky K, Rudensky AY: Foxp3-dependent microRNA155 confers competitive fitness to regulatory T cells by targeting SOCS1 protein. Immunity 2009, 30(1):80-91.

19. Kohlhaas S, Garden OA, Scudamore C, Turner M, Okkenhaug K, Vigorito E: Cutting edge: the Foxp3 target miR-155 contributes to the development of regulatory T cells. J Immunol 2009, 182(5):2578-2582.

20. Lu LF, Boldin MP, Chaudhry A, Lin LL, Taganov KD, Hanada T, Yoshimura A, Baltimore D, Rudensky AY: Function of miR-146a in controlling Treg cell-mediated regulation of Th1 responses. Cell 2010, 142(6):914-929.

21. Lewis BP, Burge CB, Bartel DP: Conserved seed pairing, often flanked by adenosines, indicates that thousands of human genes are microRNA targets. Cell 2005, 120(1):15-20.

22. Paquette J, Tokuyasu T: EGAN: exploratory gene association networks. Bioinformatics 2009, 26(2):285-286.

23. da Huang W, Sherman BT, Lempicki RA: Systematic and integrative analysis of large gene lists using DAVID bioinformatics resources. Nat Protoc 2009, 4(1):44-57.

24. Jindra PT, Bagley J, Godwin JG, lacomini J: Costimulation-dependent expression of microRNA-214 increases the ability of T cells to proliferate by targeting Pten. J Immunol 2010, 185(2):990-997.

25. Divekar AA, Dubey S, Gangalum PR, Singh RR: Dicer insufficiency and microRNA-155 overexpression in lupus regulatory T cells: an apparent paradox in the setting of an inflammatory milieu. J Immunol 2011, 186(2):924-930.

26. Li J, Wan Y, Guo Q, Zou L, Zhang J, Fang Y, Fu X, Liu H, Lu L, Wu Y: Altered microRNA expression profile with miR-146a upregulation in CD4+ T cells from patients with rheumatoid arthritis. Arthritis Res Ther 2010, 12(3):R81.

27. De Santis G, Ferracin M, Biondani A, Caniatti L, Rosaria Tola M, Castellazzi M, Zagatti B, Battistini L, Borsellino G, Fainardi E, Gavioli R, Negrini M, Furlan R, 
Granieri E: Altered miRNA expression in T regulatory cells in course of multiple sclerosis. J Neuroimmunol 2010, 226(1-2):165-171.

28. Bronevetsky Y, Villarino AV, Eisley CJ, Barbeau R, Barczak AJ, Heinz GA, Kremmer E, Heissmeyer V, McManus MT, Erle DJ, Rao A, Ansel KM: T cell activation induces proteasomal degradation of Argonaute and rapid remodeling of the microRNA repertoire. J Exp Med 2013, 210(2):417-432.

29. Ohkura N, Sakaguchi S: Treg cells acquire new directions, cytokines navigate. Immunity 2012, 37(3):443-444.

30. Zheng Y, Chaudhry A, Kas A, de Roos P, Kim JM, Chu TT, Corcoran L, Treuting P, Klein U, Rudensky AY: Regulatory T-cell suppressor program co-opts transcription factor IRF4 to control T(H)2 responses. Nature 2009, 458(7236):351-356

31. Hall AO, Beiting DP, Tato C, John B, Oldenhove G, Lombana CG, Pritchard GH, Silver JS, Bouladoux N, Stumhofer JS, Harris TH, Grainger J, Wojno ED, Wagage S, Roos DS, Scott P, Turka LA, Cherry S, Reiner SL, Cua D, Belkaid Y, Elloso MM, Hunter CA: The cytokines interleukin 27 and interferongamma promote distinct Treg cell populations required to limit infection-induced pathology. Immunity 2012, 37(3):511-523.

32. Chaudhry A, Rudra D, Treuting P, Samstein RM, Liang Y, Kas A, Rudensky AY: CD4+ regulatory $T$ cells control $T H 17$ responses in a Stat3-dependent manner. Science 2009, 326(5955):986-991.

33. Sun $L, W u J$, Yi S: Foxp3 is critical for human natural CD4 + CD25+ regulatory T cells to suppress alloimmune response. Transpl Immunol 2012, 26(2-3):71-80.

34. van Loosdregt J, Vercoulen Y, Guichelaar T, Gent YYJ, Beekman JM, van Beekum O, Brenkman AB, Hijnen DJ, Mutis T, Kalkhoven E, Prakken BJ, Coffer PJ: Regulation of Treg functionality by acetylation-mediated Foxp3 protein stabilization. Blood 2009, 115:965-974.

35. Nie H, Zheng Y, Li R, Guo TB, He D, Fang L, Liu X, Xiao L, Chen X, Wan B, Chin YE, Zhang JZ: Phosphorylation of FOXP3 controls regulatory T cell function and is inhibited by TNF-alpha in rheumatoid arthritis. Nat Med 2013, 19(3):322-328

36. Korn T, Mitsdoerffer M, Croxford AL, Awasthi A, Dardalhon VA, Galileos G, Vollmar P, Stritesky GL, Kaplan MH, Waisman A, Kuchroo VK, Oukka M: IL-6 controls Th17 immunity in vivo by inhibiting the conversion of conventional T cells into Foxp3+ regulatory T cells. Proc Natl Acad Sci U S A 2008, 105(47):18460-18465.

37. Yadav M, Huang MC, Goetzl EJ: VPAC1 (vasoactive intestinal peptide (VIP) receptor type 1) G protein-coupled receptor mediation of VIP enhancement of murine experimental colitis. Cell Immunol 2011, 267(2):124-132.

38. Gomez-Rodriguez J, Sahu N, Handon R, Davidson TS, Anderson SM, Kirby MR, August A, Schwartzberg PL: Differential expression of interleukin-17A and $-17 \mathrm{~F}$ is coupled to $\mathrm{T}$ cell receptor signaling via inducible $\mathrm{T}$ cell kinase. Immunity 2009, 31(4):587-597.

39. Yang XH, Yamagiwa S, Ichida T, Matsuda Y, Sugahara S, Watanabe H, Sato $Y$, Abo T, Horwitz DA, Aoyagi Y: Increase of CD4+ CD25+ regulatory T-cells in the liver of patients with hepatocellular carcinoma. J Hepatol 2006, 45(2):254-262

40. Zabala M, Lasarte JJ, Perret C, Sola J, Berraondo P, Alfaro M, Larrea E, Prieto J, Kramer MG: Induction of immunosuppressive molecules and regulatory $T$ cells counteracts the antitumor effect of interleukin-12-based gene therapy in a transgenic mouse model of liver cancer. J Hepatol 2007, 47(6):807-815

41. Yang P, Li QJ, Feng Y, Zhang Y, Markowitz GJ, Ning S, Deng Y, Zhao J, Jiang S, Yuan Y, Wang HY, Cheng SQ, Xie D, Wang XF: TGF-beta-miR-34a-CCL22 Signaling-Induced Treg Cell Recruitment Promotes Venous Metastases of HBV-Positive Hepatocellular Carcinoma. Cancer Cell 2012, 22(3):291-303.

42. Ueha S, Yoneyama H, Hontsu S, Kurachi M, Kitabatake M, Abe J, Yoshie O, Shibayama S, Sugiyama T, Matsushima K: CCR7 mediates the migration of Foxp3+ regulatory T cells to the paracortical areas of peripheral lymph nodes through high endothelial venules. J Leukoc Biol 2007, 82(5):1230-1238

43. Eikawa S, Ohue Y, Kitaoka K, Aji T, Uenaka A, Oka M, Nakayama E: Enrichment of Foxp3+ CD4 regulatory T cells in migrated T cells to IL-6and IL-8-expressing tumors through predominant induction of CXCR1 by IL-6. J Immunol 2010, 185(11):6734-6740.

44. Zhang N, Schroppel B, Lal G, Jakubzick C, Mao X, Chen D, Yin N, Jessberger R, Ochando JC, Ding Y, Bromberg JS: Regulatory T cells sequentially migrate from inflamed tissues to draining lymph nodes to suppress the alloimmune response. Immunity 2009, 30(3):458-469.
45. Chauhan S, Saban D, Lee H, Dana R: Levels of Foxp3 in regulatory T cells reflect their functional status in transplantation. J Immunol 2009, 182(1):148-153.

46. Joffre $O$, Santolaria T, Calise D, Saati TA, Hudrisier D, Romagnoli P, van Meerwijk JPM: Prevention of acute and chronic allograft rejection with CD4 + CD25 + Foxp3+ regulatory T lymphocytes. Nat Med 2007, 14(1):88-92.

47. Fan Z, Spencer JA, Lu Y, Pitsillides CM, Singh G, Kim P, Yun SH, Toxavidis V, Strom TB, Lin CP, Koulmanda M: In vivo tracking of 'color-coded' effector, natural and induced regulatory $T$ cells in the allograft response. Nat Med 2010, 16(6):718-722

48. Cong Y, Feng T, Fujihashi K, Schoeb TR, Elson CO: A dominant, coordinated T regulatory cell-IgA response to the intestinal microbiota. Proc Natl Acad Sci U S A 2009, 106(46):19256-19261.

49. Borsutzky S, Cazac BB, Roes J, Guzman CA: TGF-beta receptor signaling is critical for mucosal IgA responses. J Immunol 2004, 173(5):3305-3309.

50. Harrison OJ, Powrie FM: Regulatory T cells and immune tolerance in the intestine. Cold Spring Harb Perspect Biol 2013, 5(7):a018341.

51. Matharu KS, Mizoguchi E, Cotoner CA, Nguyen DD, Mingle B, Iweala Ol, McBee ME, Stefka AT, Prioult G, Haigis KM, Bhan AK, Snapper SB, Murakami H, Schauer DB, Reinecker HC, Mizoguchi A, Nagler CR: Toll-like receptor 4-mediated regulation of spontaneous helicobacter-dependent colitis in IL-10-deficient mice. Gastroenterology 2009, 137(4):1380-1390. e1383.

52. Forward NA, Furlong SJ, Yang Y, Lin TJ, Hoskin DW: Signaling through TLR7 enhances the immunosuppressive activity of murine CD4 + CD25+ T regulatory cells. J Leukoc Biol 2009, 87(1):117-125.

53. Nyirenda MH, Sanvito L, Darlington PJ, O'Brien K, Zhang GX, Constantinescu CS, Bar-Or A, Gran B: TLR2 stimulation drives human naive and effector regulatory $T$ cells into a Th17-like phenotype with reduced suppressive function. J Immunol 2011, 187(5):2278-2290.

54. Zhang Y, Luo F, Cai Y, Liu N, Wang L, Xu D, Chu Y: TLR1/TLR2 agonist induces tumor regression by reciprocal modulation of effector and regulatory T cells. J Immunol 2011, 186(4):1963-1969.

doi:10.1186/1471-2407-14-489

Cite this article as: Chen et al.: Special role of Foxp3 for the specifically altered microRNAs in Regulatory T cells of HCC patients. BMC Cancer 2014 14:489.

\section{Submit your next manuscript to BioMed Central and take full advantage of:}

- Convenient online submission

- Thorough peer review

- No space constraints or color figure charges

- Immediate publication on acceptance

- Inclusion in PubMed, CAS, Scopus and Google Scholar

- Research which is freely available for redistribution

Submit your manuscript at www.biomedcentral.com/submit
C Biomed Central 